\title{
Remoção de tórus mandibular bilateral com finalidade protética: relato de caso clínico
}

\author{
Removal of bilateral mandibular tórus with protetic purpose: clinical case report \\ Extracción de toro mandibular bilateral con fin protésico: reporte de caso clínico
}

Recebido: 04/03/2021 | Revisado: 12/03/2021 | Aceito: 16/03/2021 | Publicado: 23/03/2021

Marcelo Wiler Gomes da Silva

ORCID: https://orcid.org/0000-0002-3416-8333

Faculdade Patos de Minas, Brasil

E-mail: marcelowiler@gmail.com

André Luís Oliveira Garcia

ORCID: https://orcid.org/0000-0002-8874-5394

Faculdade Patos de Minas, Brasil

E-mail: andreluisoliveiragarcia@hotmail.com

Lia Dietrich

ORCID: https://orcid.org/0000-0001-7887-8591

Clínica Privada, Brasil

E-mail: lia_dietrich@yahoo.com.br

Lilian de Barros

ORCID: https://orcid.org/0000-0002-9204-3539

Clínica Privada, Brasil

E-mail: lilidebarros@hotmail.com

Henrique Cury Viana

ORCID: https://orcid.org/0000-0001-6168-0917

Faculdade Patos de Minas, Brasil

E-mail: curyhenrique@hotmail.com

Pedro Henrique Justino Oliveira Limirio

ORCID: https://orcid.org 0000-0002-0089-3772

Universidade Federal de Uberlândia, Brasil

E-mail: pedro_hjlo@ hotmail.com

Marcelo Dias Moreira de Assis Costa

ORCID: https://orcid.org/0000-0001-9148-3674

Universidade Federal de Uberlândia, Brasil

E-mail: marcelodmac@yahoo.com.br

\begin{abstract}
Resumo
O tórus mandibular pode ser definido como uma exostose comum e assintomática, que normalmente não necessita de tratamento, exceto em situações específicas. Uma das indicações para tal remoção é para adaptação de próteses removíveis. Nesses casos é realizada a cirurgia para garantir melhor estabilidade e função a prótese que será instalada futuramente. Este artigo foi construído por meio da realização de um caso clínico, que apresentou como principal objetivo a remoção de um tórus mandibular bilateral com finalidade de permitir a confecção de uma prótese parcial removível.
\end{abstract}

Palavras-chave: Prótese removível; Tórus mandibular; Cirurgia bucal.

\begin{abstract}
The mandibular torus can be defined as a common and asymptomatic exostosis, which normally does not require treatment, except in specific situations. One of the indications for such removal is for adaptation of removable prostheses. In these cases surgery is performed to ensure better stability and function the prosthesis that will be installed in the future. This article was constructed by means of the accomplishment of a clinical case, that had as main objective the removal of a bilateral mandibular torus with the purpose of allowing the preparation of a partial removable prosthesis.

Keywords: Removable prosthesis; Torus mandibular; Surgery oral.

\section{Resumen}

El toro mandibular se puede definir como una exostosis frecuente y asintomática, que normalmente no requiere tratamiento, salvo en situaciones específicas. Una de las indicaciones para dicha extracción es adaptar prótesis removibles. En estos casos, se realiza una cirugía para asegurar una mejor estabilidad y función de la prótesis que se instalará en el futuro. Este artículo fue construido a través de la realización de un caso clínico, que tenía como
\end{abstract}


principal objetivo la extracción de un toro mandibular bilateral para permitir la fabricación de una prótesis parcial removible.

Palabras clave: Prótesis removible; Toro mandibular; Cirugía oral.

\section{Introdução}

O tórus mandibular é uma exostose comum que se desenvolve ao longo da superfície lingual da mandíbula, assim como o tórus palatino. Sua causa é multifatorial, podendo ser de origem genética, relacionado a fatores ambientais, como o estresse mastigatório, distúrbios nutricionais, processo contínuo de desenvolvimento e hábitos parafuncionais. Algumas autoridades sugerem que o tórus é herdado como um traço autossômico dominante (Bruce et al., 2004).

Sua ocorrência é predominantemente bilateral, aproximadamente $90 \%$ dos casos (Bruce et al., 2004). Podem aparecer em radiografias periapicais dependendo do tamanho e nas radiografias oclusais são facilmente visualizados. Apresenta-se em forma circunscrita, tendo de $1 \mathrm{~cm}$ a $5 \mathrm{~cm}$, sendo composto por osso hiperplásico de estrutura compacta e uma parte central trabecular associado a medula gordurosa (García-García et al., 2010). O exame radiográfico é imprescindível para o diagnóstico diferencial, já que o aumento volumétrico notado pelo exame clinico e palpação podem ser formação de abscessos, neoplasias ósseas, neoplasias de glândulas salivares e tumor vascular (García-García et al., 2010; James Jordan, 2013).

Normalmente essa exostose apresenta-se assintomática, exceto em casos onde a mucosa que os recobre seja fina e ulcere em razão do traumatismo secundário. Geralmente não necessitam de tratamento cirúrgico por não promoverem grande interferência fisiológica. A remoção cirúrgica está indicada em casos de dor, interferência na mastigação, fonação e para estabilidade de prótese parcial removível ou prótese total (Ghahremani et al., 2020). Após a remoção cirúrgica, o conteúdo removido deve ser encaminhado para a biópsia. Sendo confirmado pelas características histopatológicas onde observa-se uma massa de osso cortical lamelar denso, sendo algumas vezes notada uma zona interna de osso trabecular semelhante ao osso normal (Bruce et al., 2004; Ghahremani et al., 2020).

Nas cirurgias pré protéticas a remoção é indicada para melhor adaptação da prótese removível que será posteriormente confeccionada e instalada. Desta forma a retenção e estabilidade estarão garantidas e as ulcerações durante a reabilitação mastigatória serão reduzidas ou inexistentes (Ghahremani et al., 2020).

Este trabalho visa relatar a remoção do torus mandibular para posterior confecção de prótese removível, recorrendo a apresentação de um caso clínico de torus mandibular bilateral. Tal caso foi analisado fatores etiológicos, características clínicas e radiográficas, diagnóstico, necessidade de remoção e características histopatológicas.

\section{Metodologia}

Segundo Pereira, Shitsuka, Parreira e Shitsuka (2018) é um estudo qualitativo, descritivo, retrospectivo, referente a um assunto específico, detalhado para destacar suas particularidades. Esse trabalho foi submetido e aprovado pelo Comitê de Ética em Pesquisa da Faculdade Patos de Minas - FPM sob o número parecer 2.662.602 e CAAE - 88756418.6.0000.8078.

\section{Relato de Caso}

Paciente sexo masculino, 47 anos de idade, compareceu a Policlínica da Faculdade Patos de Minas - FPM relatando insatisfação com seu sorriso. Inicialmente foi realizada a triagem e anamnese do paciente. Em seguida a revisão da história médica e o exame clínico. Como parte do tratamento odontológico necessário, foi submetido a um exame mais detalhado dos rebordo alveolares. Nesse momento foi observado uma massa de consistência endurecida, lobular, na região de processo alveolar lingual da mandíbula, recoberta por mucosa de aparência normal. Paciente não relatava nenhum desconforto ou dor com a presença da massa, ao qual nunca havia reparado anteriormente (Figura 1). Para elucidação diagnóstica foi realizado 
exames radiográficas no local, que apresentava imagem compatível com osso cortical (Figura 2). Foi fechado assim o diagnóstico de Torus Mandibular Bilateral. Devido a necessidade de confecção de uma prótese parcial removível com uma barra lingual o torus impediria sua confecção, necessitando assim sua remoção cirúrgica.

Figura 1 - Aspecto Clínico.

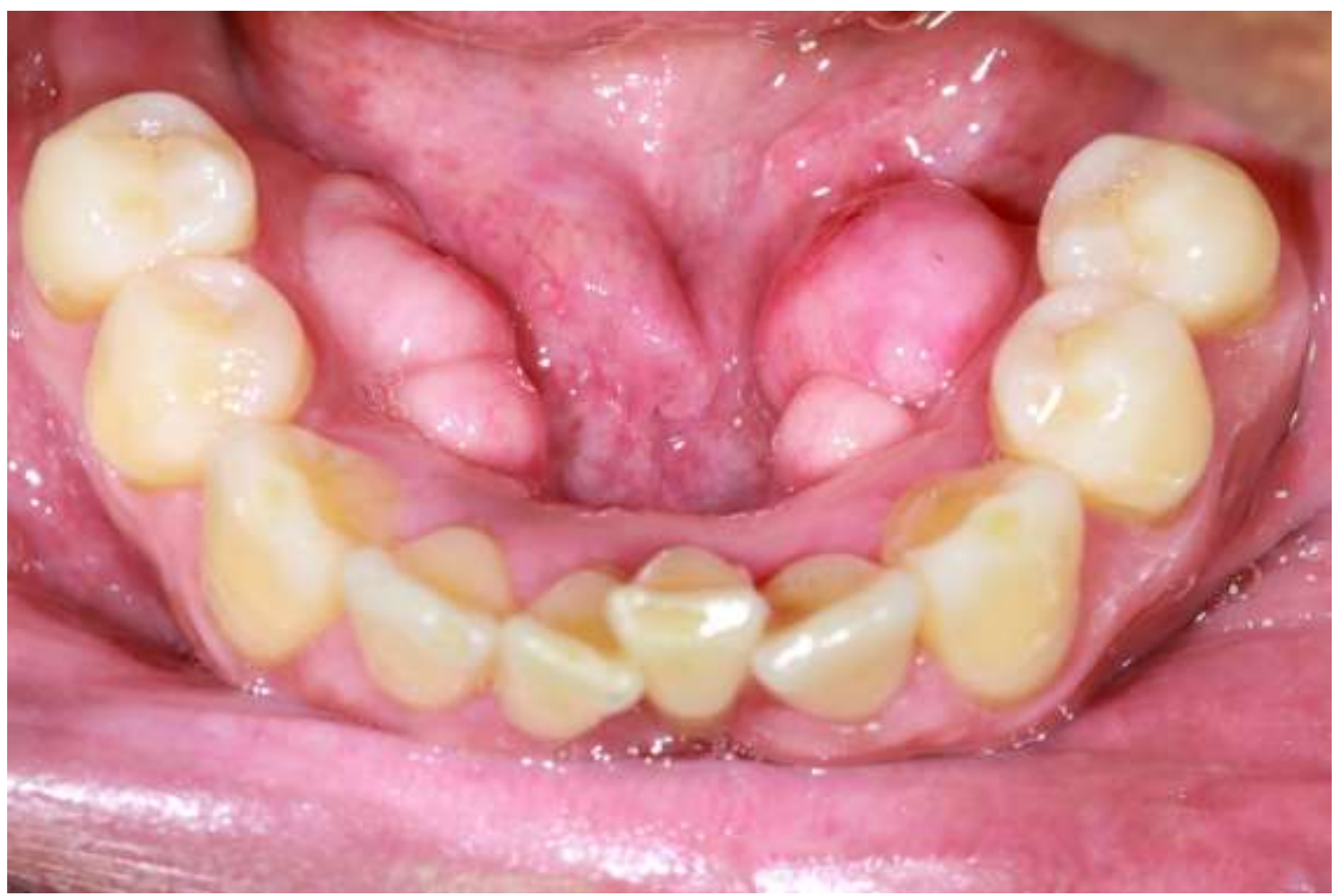

Fonte: Autores (2020).

Figura 2 - Aspecto Radiográfico.

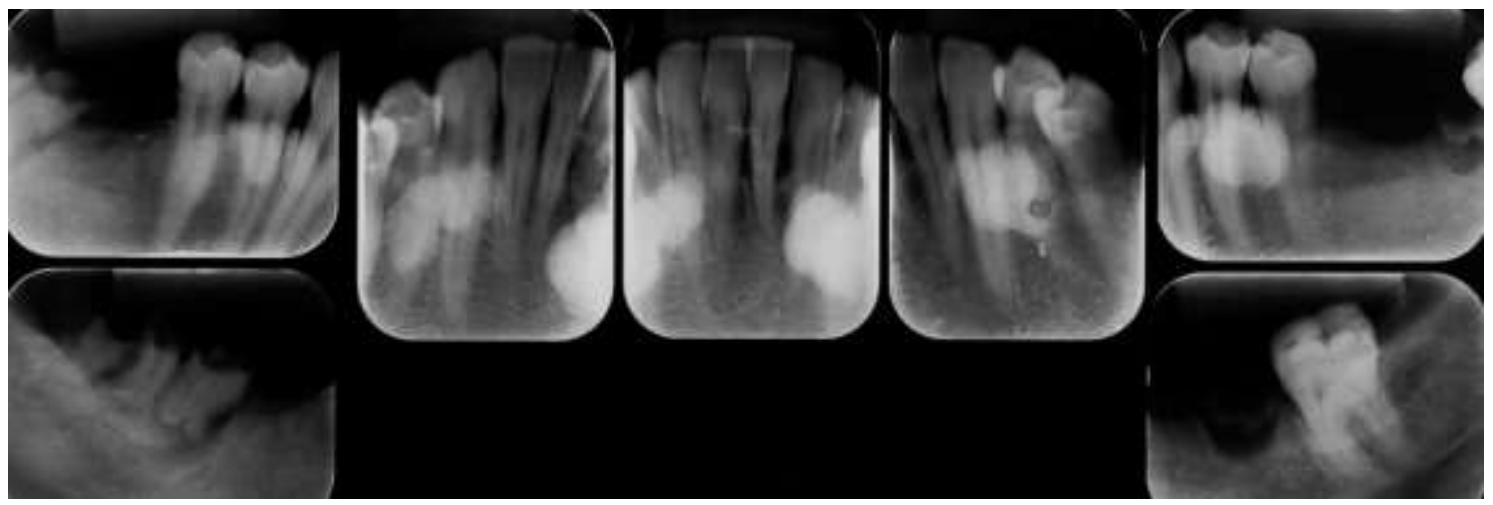

Fonte: Autores (2020).

O procedimento de remoção do torus foi realizada sob anestesia por bloqueio regional bilateral dos nervos alveolar inferior e lingual, associado a infiltrações terminais para hemostasia. O anestésico de escolha foi lidocaína a $2 \%$ associada a epinefrina 1:100.000. Foram utilizados 4 tubetes anestésicos.

Uma incisão sulcular na região lingual dos dentes inferiores associada a uma extensão para o rebordo ósseo alveolar edêntulo posterior de ambos os lados foi realizada seguido pelo descolamento mucoperiosteal deixando uma faixa de tecido inserida á linha média entre os limites anteriores das incisões para evitar hematomas (Figura 3). 
Figura 3 - Aspecto Trans-cirúrgico.

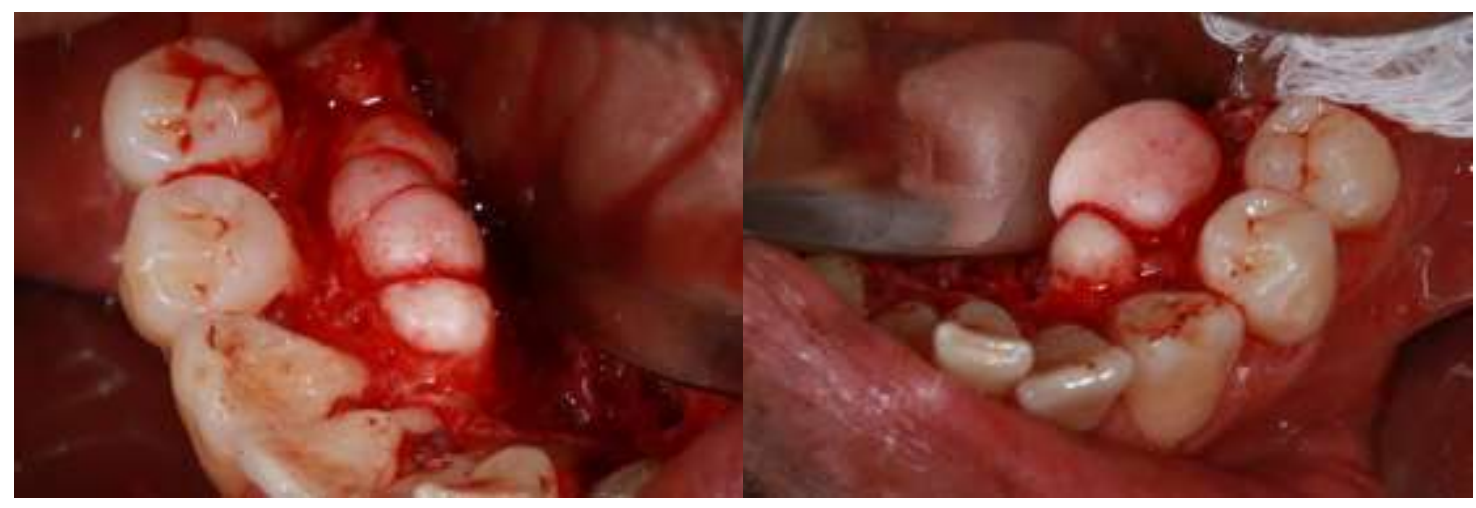

Fonte: Autores (2020).

Uma canaleta óssea foi confeccionada no limite interno da cortical lingual da mandíbula com uma broca Zekrya e em seguida foi utilizado o descolador de Molt para fraturar o fragmento do torus (Figura 4).

Figura 4 - Confecção da canaleta e clivagem óssea.

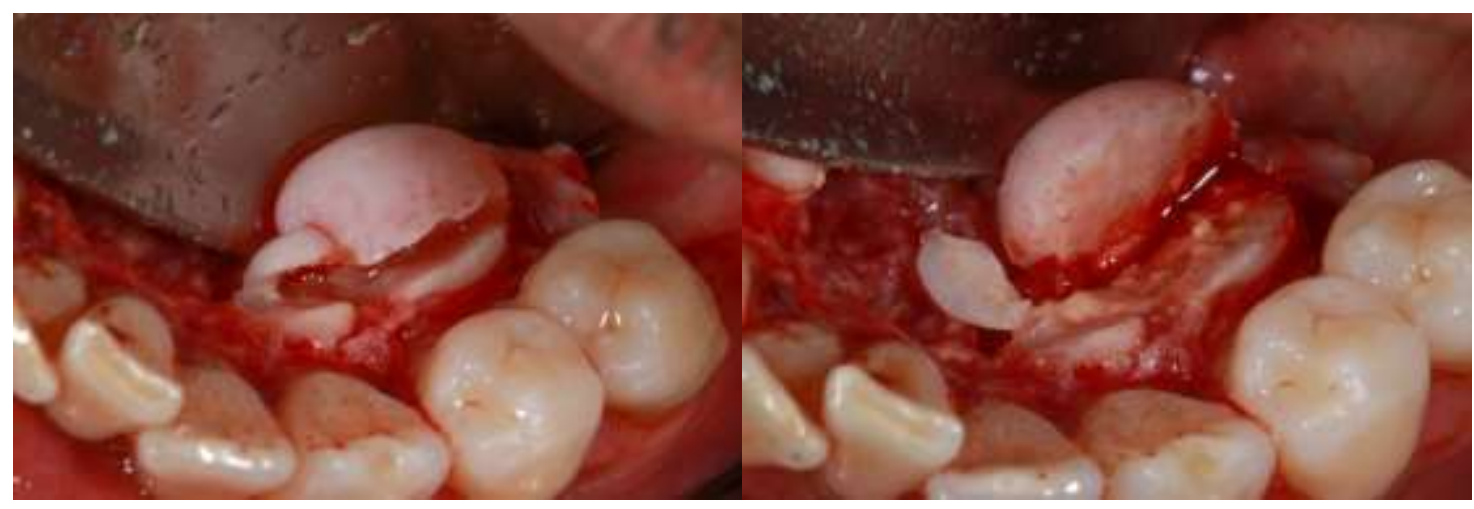

Fonte: Autores (2020).

O aplainamento foi realizado com uma broca Maxicutt em peça reta (Figura 5), para correção do formato do rebordo alveolar para uma melhor adaptação da futura prótese. Após uma irrigação copiosa da região e conferência da hemostasia (Figura 6), os tecidos foram suturados com fio de nylon por meio de sutura simples. 
Research, Society and Development, v. 10, n. 3, e48410313564, 2021

(CC BY 4.0) | ISSN 2525-3409 | DOI: http://dx.doi.org/10.33448/rsd-v10i3.13564

Figura 5 - Aplainamento Ósseo.

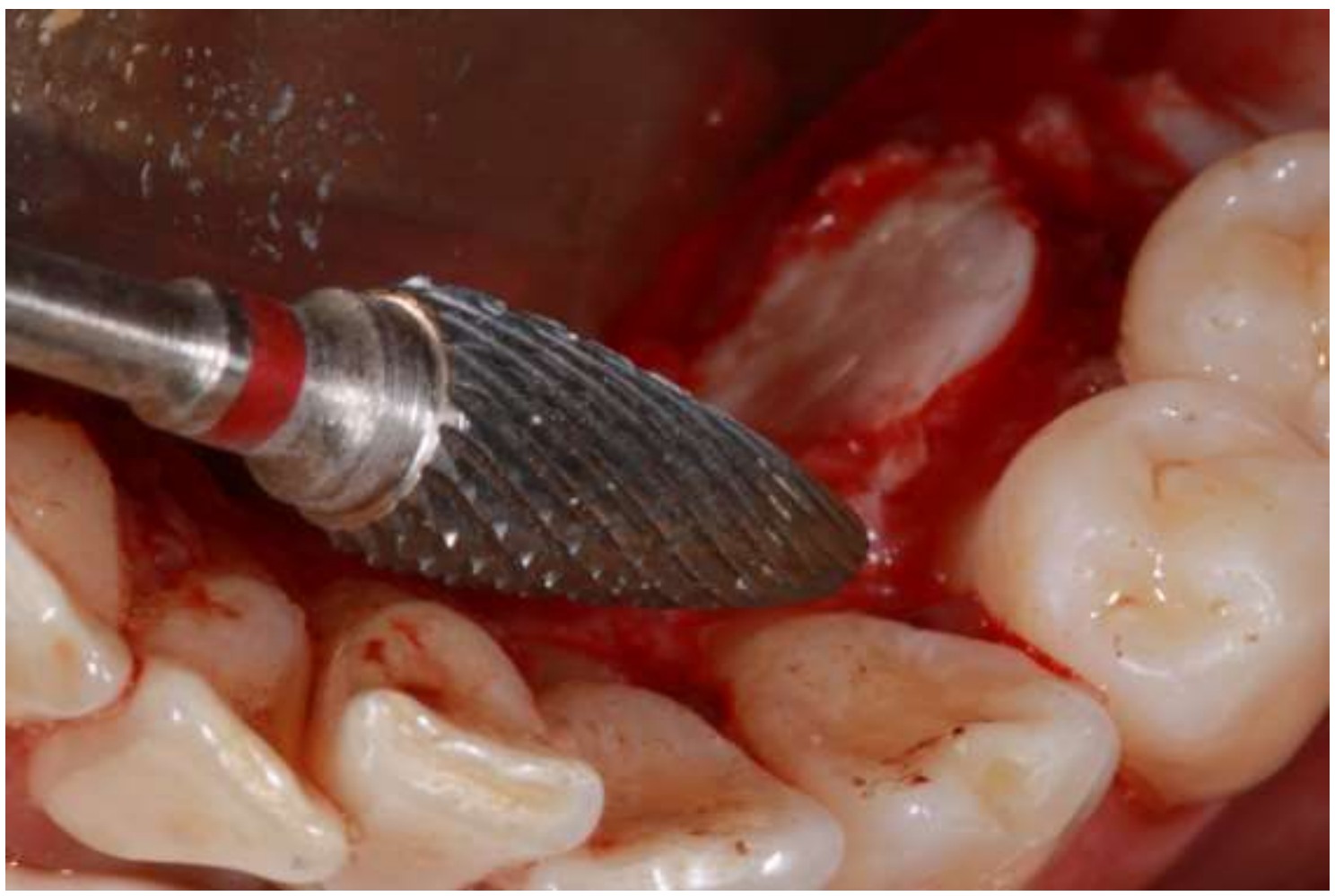

Fonte: Autores (2020).

Figura 6 - Aspecto Cirúrgico Final.

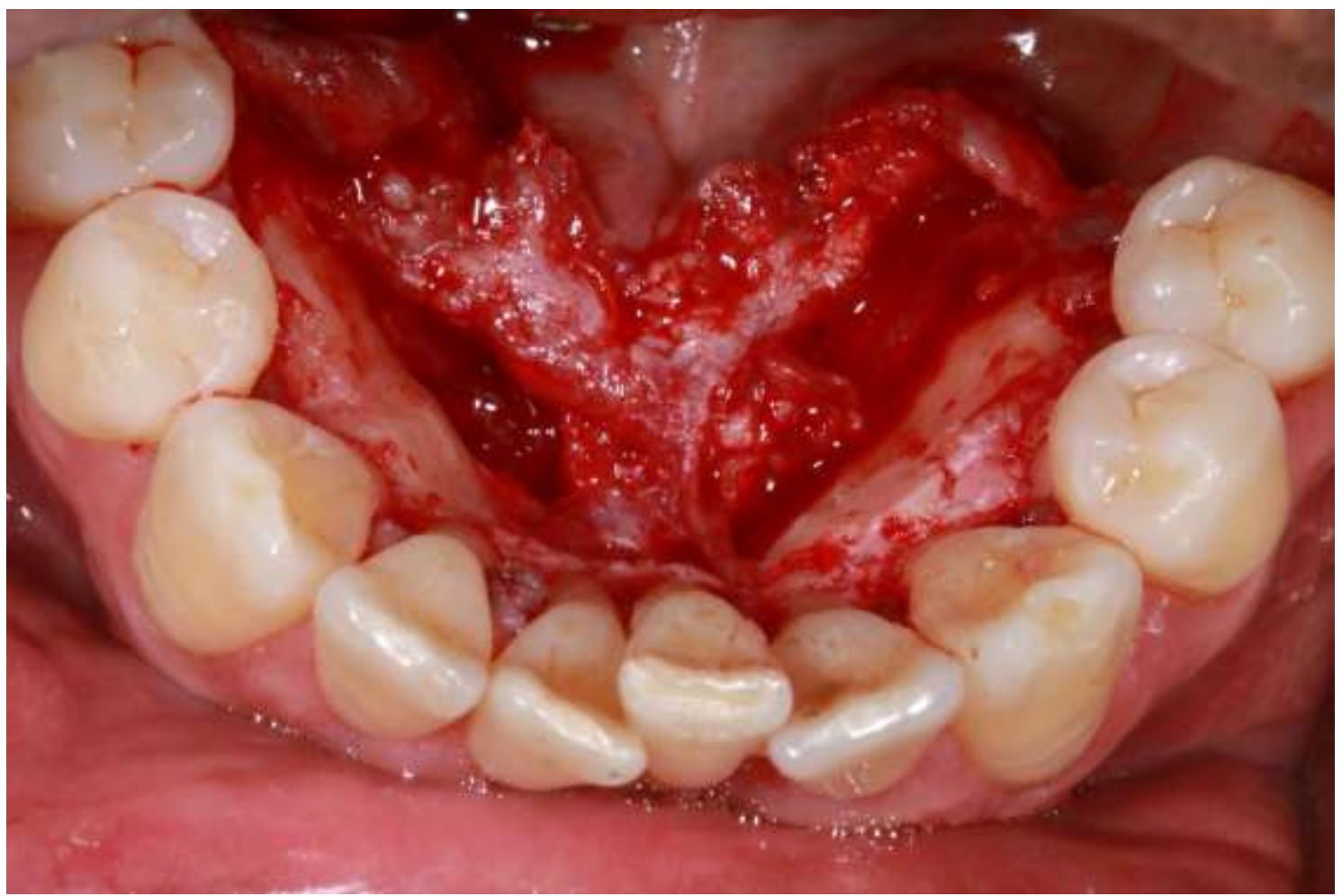

Fonte: Autores (2020).

A exostose removida foi enviada para biopsia e o resultado do exame histopatológico foi compatível com osso de aspecto normal, assim fechando o diagnóstico de Tórus Mandibular. 
Após 07 dias de pós-operatório foi observado cicatrizaçnao satisfatória da ferida cirúrgica e então foi realizada a remoção das suturas. Após período de remodelação e estabilização dos tecidos, aproximadamente 90 dias, o paciente foi encaminhado para confecção da prótese parcial removível. O paciente seguem em acompanhamento clínico sem intercorrências (Figura 7).

Figura 7 - Aspecto Clínico da região após 3 meses de acompanhamento.

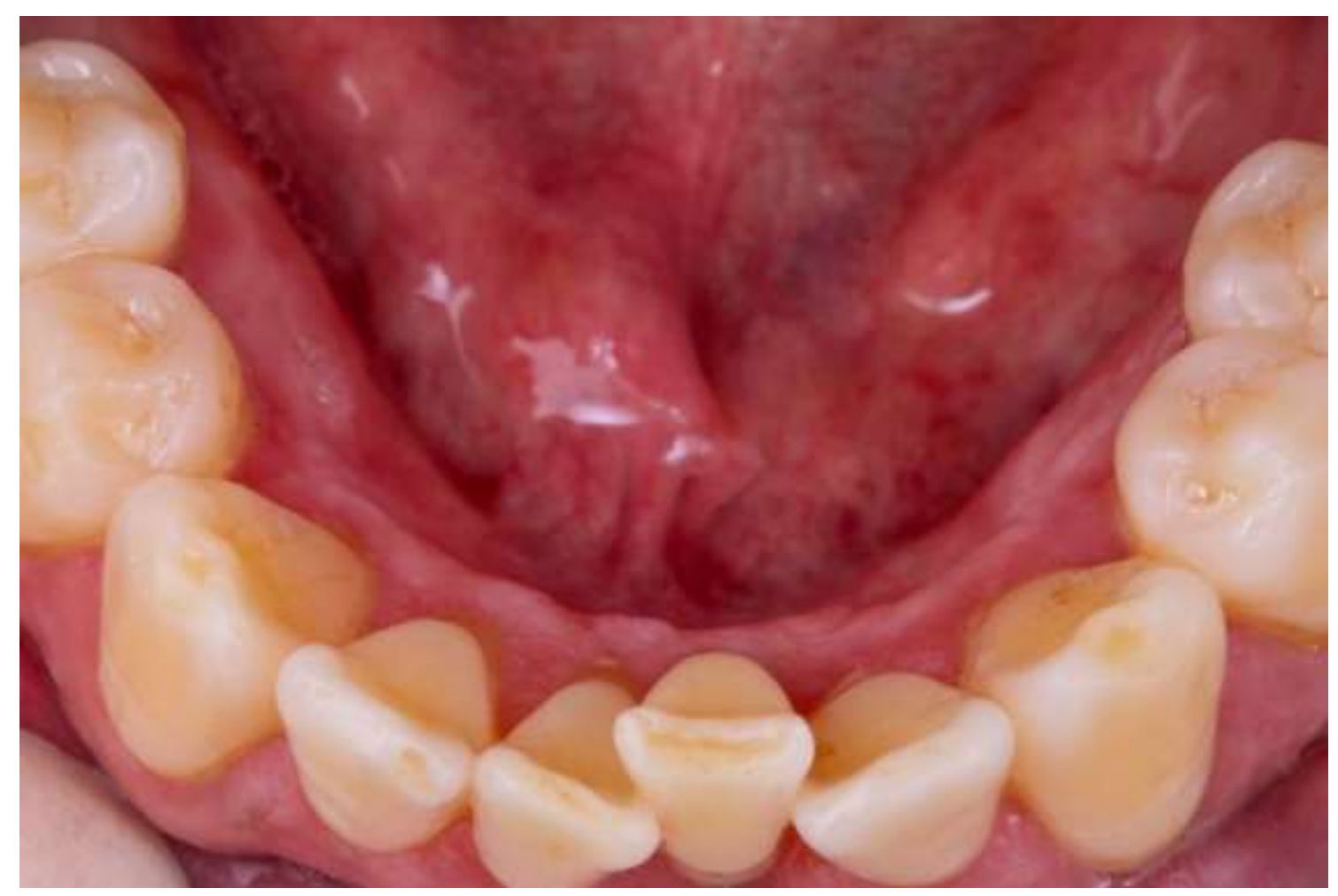

Fonte: Autores (2020).

\section{Discussão}

O torus mandibular, palavra derivada do latim torus, pode ser definido como uma exostose, ou seja, um crescimento ósseo protuberante, em forma circular e localizado, de caráter benigno, situando-se na superfície óssea cortical, sobre a linha miloióidea na região dos pré-molares (Al-Bayaty et al., 2001; Bruce et al., 2004). Chao et al. (2015) relata que o torus mandibular é principalmente observado na região lingual do osso mandibular em pacientes de meia-idade, não necessariamente na região de pré molares (Chao et al., 2015).

$\mathrm{Na}$ maioria dos casos, o achado é geralmente acidental e observado durante o exame clínico no consultório odontológico. Isso porque eles são assintomáticos em grande parte e aqueles que os têm nem sempre estão cientes disso. Às vezes, os pacientes podem apresentar distúrbios fonatórios, limitação da mecânica mastigatória, ulcerações da mucosa, depósitos de alimentos, instabilidade protética, e alguns pacientes podem experimentar a cancerofobia e consultar um profissional para procurar uma solução (Al-Bayaty et al., 2001, p.).

O crescimento do torus é gradual, sendo maior na segunda ou terceira década de vida. Quanto a esta seção, entre os estudos revisados, não há consenso sobre como classificar o crescimento; cada estudo classifica o crescimento de forma diferente (Bruce et al., 2004). Apresentam tamanho variando entre 3 e $4 \mathrm{~cm}$, mas normalmente possuem diâmetro menor que $1,5 \mathrm{~cm}$. Sua ocorrência pode ser unilateral ou bilateral, sendo a segunda predominante, ocorrendo em $90 \%$ dos pacientes acometidos. Apresenta como características crescimento lento e progressivo, ausência de sintomatologia dolorosa na maioria 
dos casos (com exceção aqueles em que a mucosa que os recobre apresenta-se lesionada devido a traumatismos), maior incidência em jovens e predileção pelo sexo feminino (Al-Bayaty et al., 2001).

Várias causas possíveis foram discutidas para explicar a etiologia do torus mandibular, mas o modelo aceito para a formação dessas protuberâncias ósseas ainda está em questão. Historicamente, o foco dominante está na genética, e a hereditariedade foi analisada usando estudos familiares estudos regionais, ou comparando grupos étnicos (Al-Bayaty et al., 2001, p.; Johnson et al., 1965; Simunković et al., 2011). O desenvolvimento do torus mandibular pode ser devido a fatores genéticos, incluindo gênero e etnia do paciente; fatores ambientais, como a taxa de sobrevivência dos dentes e desnutrição; ou fatores funcionais, como mastigação traumática (Cortes et al., 2014). Em relação aos fatores etiológicos que levam ao aparecimento do toro os mais frequentemente aceitos são os hábitos parafuncionais, os fatores ambientais e a predisposição genética. Alterações como o bruxismo e o estresse mastigatório são fortemente associadas a tal crescimento ósseo (Nascimento Filho et al., 2004).

O diagnóstico diferencial é obtido primeiramente através do exame radiográfico, já que clinicamente tal aumento volumétrico tem características confundidas com abcessos, neoplasias de glândulas salivares, neoplasias ósseas, tumores vasculares e até mesmo dentes inclusos. Dependendo de sua extensão, podem ser encontrados em radiografias panorâmicas e até em radiografias periapicais. As imagens radiográficas apresentam áreas circunscritas sobrepostas pela imagem radiopaca das raízes dos dentes inferiores (James \& Jordan, 2013). O exame histopatológico revela que é semelhante à estrutura compacta do osso normal, possuindo uma estrutura ligeiramente esponjosa com espaços medulares (García-García et al., 2010).

A remoção do torus nem sempre é necessária. A causa mais frequente de extirpação continua sendo a necessidade de tratamento protético ou de ser uma fonte potencial de osso cortical autógeno para enxertos em cirurgia periodontal, cirurgia de cisto ou cirurgia de implante, embora a estabilidade a longo prazo dos enxertos seja incerta (Fragoso et al., 2020; Ganz, 1997; Sonnier et al., 1999). Grandes toros podem ser removidos, especialmente se forem um obstáculo para o tratamento protético (García-García et al., 2010). Em casos onde a fisiologia oral seja alterada, comprometendo e alterando a deglutição, a dicção e a fonação, ou ainda em casos onde os traumas na mucosa que recobre a protuberância sejam frequentes, gerando ulcerações dolorosas, recomenda-se a extirpação. Em casos nos quais a reabilitação oral do paciente inclui próteses totais ou parciais removíveis, existe também há necessidade da retirada desse crescimento ósseo, já que o mesmo compromete a adaptação e a estabilidade das peças protéticas (Ghahremani et al., 2020; Paula et al., 2010).

Quando é necessária a remoção do toro existe uma variedade de técnicas cirúrgicas que podem ser empregadas. Podemos dividi-las em dois tipos: as que utilizam instrumento rotatório e as que não utilizam. Alguns autores defendem o uso de instrumento rotatório apenas para toros pequenos, sendo o cinzel e o martelo os instrumentos de escolha nos demais casos. Já outros veem o rotatório como necessidade apenas para regularização óssea após a retirada da protuberância (Goncalves et al., 2013). Em alguns casos é defendido o uso do rotatória para remoção total do toro, devolvendo a mandíbula seu aspecto normal (Sonnier et al., 1999). Outra técnica sugerida consiste na criação de sulcos de orientação com o rotatório e em seguida a remoção da lesão com martelo e cinzel, tendo a canaleta criada como apoio, facilitando a extirpação e evitando complicações pós cirúrgicas (Goncalves et al., 2013).

Vale ressaltar que em casos onde os instrumentos rotatórios são empregados é necessária a irrigação abundante com soro fisiológico. Devido aos movimentos a broca aquece, podendo levar o tecido a necrose induzida por calor. Sendo realizada a irrigação em sua ponta ativa, é induzido o resfriamento da mesma, evitando lesionar ou necrosar o tecido (Sverzut et al., 2009).

Após a remoção cirúrgica, o material removido deve ser encaminhado para a biopsia de modo a se confirmar o diagnóstico por meio do exame histopatológico. Nos aspectos histopatológicos, a estrutura do toro assemelha-se ao osso 
mandibular, sendo composto de osso esponjoso com espaços medulares recoberto por osso compacto (Karaca et al., 2019).

A cirurgia para remoção do toro, assim como qualquer outra cirurgia odontológica, requer cuidados pós-operatórios. $\mathrm{O}$ controle da dor é realizado por meio de analgésicos e anti-inflamatórios, a antibioticoterapia também é realizada. Como analgésico de escolha temos a dipirona sódica, devido a sua absorção e rápida ação. No caso dos anti-inflamatórios opta-se principalmente pelos não esteroides, como o ibuprofeno e a nimesulida, seu mecanismo de ação e a associação positiva com analgésico levam a essa escolha. No caso dos antibióticos a amoxicilina é amplamente utilizada devido a sua capacidade de combater bactérias oportunistas que se alojam na orofaringe (Ganz, 1997).

\section{Conclusão}

Conclui-se então que a remoção do toros mandibular bilateral foi efetiva para a melhor adaptação da prótese que futuramente será instalada. Desta forma ficam garantidas uma melhor adaptação, estabilidade e retenção da peça protética, auxiliando na reabilitação funcional e estética, evitando assim, evitando ulcerações por trauma.

\section{Referências}

Al-Bayaty, H. F., Murti, P. R., Matthews, R., \& Gupta, P. C. (2001). An epidemiological study of tori among 667 dental outpatients in Trinidad \& Tobago, West Indies. International Dental Journal, 51(4), 300-304. https://doi.org/10.1002/j.1875-595x.2001.tb00842.x

Bruce, I., Ndanu, T. A., \& Addo, M. E. (2004). Epidemiological aspects of oral tori in a Ghanaian community. International Dental Journal, 54(2), 78-82. https://doi.org/10.1111/j.1875-595x.2004.tb00259.x

Chao, P.-J., Yang, H.-Y., Huang, W.-H., Weng, C.-H., Wang, I.-K., Tsai, A. I., \& Yen, T.-H. (2015). Oral Tori in Chronic Hemodialysis Patients. BioMed Research International, 2015. https://doi.org/10.1155/2015/897674

Cortes, A. R. G., Jin, Z., Morrison, M. D., Arita, E. S., Song, J., \& Tamimi, F. (2014). Mandibular tori are associated with mechanical stress and mandibular shape. Journal of Oral and Maxillofacial Surgery: Official Journal of the American Association of Oral and Maxillofacial Surgeons, 72(11), 2115-2125. https://doi.org/10.1016/j.joms.2014.05.024

Fragoso, L. N. M., Silva, R. M. da, Flores, N. da C., Lucena, A. L. de M., Florentino, V. G. B., Neto, L. F. de A., Monteiro, R. V. T., Freitas, G. B. de, Filho, J. C. W. P. de A., \& Rocha, J. F. (2020). Utilização de tórus mandibular para reconstrução parcial de rebordo atrófico em mandíbula-Relato de caso clínico. Research, Society and Development, 9(7), e537974412-e537974412. https://doi.org/10.33448/rsd-v9i7.4412

Ganz, S. D. (1997). Mandibular tori as a source for onlay bone graft augmentation: A surgical procedure. Practical Periodontics and Aesthetic Dentistry: PPAD, 9(9), 973-982; quiz 984.

García-García, A. S., Martínez-González, J.-M., Gómez-Font, R., Soto-Rivadeneira, A., \& Oviedo-Roldán, L. (2010). Current status of the torus palatinus and torus mandibularis. Medicina Oral, Patologia Oral Y Cirugia Bucal, 15(2), e353-360.

Ghahremani, G. G., Naimi, D. R., \& Ghahremani, Z. K. (2020). Torus Lesions of the Jaw: Diagnosis and Clinical Implications. International Journal of Clinical Practice, e13697. https://doi.org/10.1111/ijcp.13697

Goncalves, T. M. S. V., de Oliveira, J. A., Sanchez-Ayala, A., \& Rodrigues Garcia, R. C. M. (2013). Surgical resection and prosthetic treatment of an extensive mandibular torus. General Dentistry, 61(1), 65-68.

James, J., Jordan, R. C. K., Regezi. (2013). Patologia Oral. Correlações Clinicopatológicas (6ª ed.). Elsevier.

Johnson, C. C., Gorlin, R. J., \& Anderson, V. E. (1965). Torus Mandibularis: A Genetic Study. American Journal of Human Genetics, $17(5)$, $433-442$.

Karaca, I. R., Ozturk, D. N., \& Akinci, H. O. (2019). Mandibular Torus Harvesting for Sinus Augmentation: Two-Year Follow-Up. Journal of Maxillofacial and Oral Surgery, 18(1), 61-64. https://doi.org/10.1007/s12663-018-1135-y

Nascimento Filho, E., Seixas, M. T., Mazzoni, A., \& Weckx, L. L. M. (2004). Multiple exophytic osteomas of craniofacial bones not associated with Gardner's Syndrome: A case report. Revista Brasileira de Otorrinolaringologia, 70(6), 836-839. https://doi.org/10.1590/S0034-72992004000600023

Paula, J. S. de, Rezende, C. de C., \& Paula, M. V. Q. de. (2010). Torus mandibular. Odonto, 18(35), 81-86. https://doi.org/10.15603/21761000/odonto.v18n35p81-86

Pereira, A. S., Shitsuka, D. M., Parreira, F. J., \& Shitsuka, R. (2018). Metodologia do trabalho científico. UFSM. https://repositorio.ufsm.br/bitstream/handle/ 1/15824/Lic_Computacao_Metodologia-Pesquisa-Cientifica.pdf.

Simunković, S. K., Bozić, M., Alajbeg, I. Z., Dulcić, N., \& Boras, V. V. (2011). Prevalence of torus palatinus and torus mandibularis in the Split-Dalmatian County, Croatia. Collegium Antropologicum, 35(3), 637-641.

Sonnier, K. E., Horning, G. M., \& Cohen, M. E. (1999). Palatal tubercles, palatal tori, and mandibular tori: Prevalence and anatomical features in a U.S. population. Journal of Periodontology, 70(3), 329-336. https://doi.org/10.1902/jop.1999.70.3.329 
Research, Society and Development, v. 10, n. 3, e48410313564, 2021

(CC BY 4.0) | ISSN 2525-3409 | DOI: http://dx.doi.org/10.33448/rsd-v10i3.13564

Sverzut, C. E., Trivellato, A. E., Sverzut, A. T., Luna, A. H. B., Santos, T. I., \& Pontes, C. de B. (2009). Rehabilitation of severely resorbed edentulous mandible using the modified visor osteotomy technique. Brazilian Dental Journal, 20(5), 419-423. https://doi.org/10.1590/S0103-64402009000500011 